\section{Should All Patients with Type 2 Diabetes Mellitus and Cardiovascular Disease Receive an SGLT2 Inhibitor?}

\section{THE "PRO" SIDE}

Innovations - ordering groceries online, moving your files to cloud storage, using an app to have a stranger give you a ride - are often approached with uncertainty. Healthy skepticism is useful, particularly for new drugs. In type 2 diabetes mellitus, the story of the ill-fated drug rosiglitazone serves as a cautionary tale: new does not necessarily equal better. In response, the US Food and Drug Administration mandated that pharmaceutical manufacturers be required to conduct randomized controlled trials with the goal of establishing cardiovascular safety for all new antidiabetic drugs. ${ }^{1}$ Two recent trials (EMPA-REG OUTCOME ${ }^{2}$ and CANVAS $^{3}$ ) have demonstrated that sodium glucose co-transporter 2 (SGLT2) inhibitors, a novel class of medications that promote glycosuria by inhibiting glucose reabsorption in the proximal convoluted tubule independent of insulin secretion, are not only safe from a cardiovascular perspective, but actually reduce the risk of meaningful cardiovascular outcomes. The "flozins", as they are known, have purported health benefits beyond glycemic control, including weight loss, reduction in blood pressure, and increase in high-density lipoprotein cholesterol. ${ }^{4}$ SGLT2 inhibitors should be utilized with watchful optimism, as the observed cardiovascular benefit is nontrivial. However, enthusiasm for these agents is being tempered with appropriate trepidation — no one wants to get "rosiglitazoned" again.

The affirmative in this debate can be distilled into 2 key arguments: first, SGLT2 inhibitors have reduced clinically meaningful cardiovascular outcomes in multiple large randomized controlled trials, and second, this evidence stands alone, given that for most other antidiabetic drugs there is an appalling lack of cardiovascular benefit.

The EMPA-REG OUTCOME trial compared empagliflozin with placebo in 7020 patients with type 2 diabetes and established cardiovascular disease. ${ }^{2}$ After 3.1 years, the primary composite outcome of cardiovascular death, nonfatal myocardial infarction, or nonfatal stroke was significantly lower with empagliflozin, for a number needed to treat (NNT) of 63. As well, empagliflozin significantly reduced all-cause death (NNT 39), cardiovascular death (NNT 46), serious adverse events (NNT 53), hospital admissions for heart failure (NNT 72), and incident/worsening nephropathy (NNT 17). 2,5,6 The CANVAS trial compared canagliflozin with placebo in 10142 patients with type 2 diabetes who had established, or were at high risk of, cardiovascular disease. $^{3}$ After 3.6 years, the primary composite outcome (which was the same as in the EMPA-REG OUTCOME study) was significantly lower with canagliflozin, for an NNT of 61. However, all-cause and cardiovascular death were not significantly reduced with treatment. Hospital admissions for heart failure (NNT 87), adverse renal outcomes (NNT 80), and serious adverse events (NNT 18) were significantly lower with canagliflozin. Adverse events associated with SGLT2 inhibitor therapy included genital infections (number needed to harm [NNH] 6-14 for women and 12-29 for men), as well as volume depletion (NNH 38), amputations (NNH 96), and fractures (NNH 286), the latter 3 of which were associated only with canagliflozin. Despite these adverse events, it is reasonable to conclude that the potential cardiovascular benefit far exceeds the potential harm. Although one cannot draw firm conclusions from indirectly comparing 2 different trials, it appears that empagliflozin has more favourable evidence than canagliflozin.

This evidence is exceptional when compared with the evidence for other antihyperglycemic agents. Sulfonylureas have been associated with increased cardiovascular events in observational trials, whereas a neutral effect has been observed in randomized controlled trials. ${ }^{7}$ Regardless, there is no robust evidence suggesting even a glimpse of cardiovascular benefit. Among the thiazolidinediones, rosiglitazone was associated with an increased risk of myocardial infarction, ${ }^{8}$ and in the PROactive trial, pioglitazone reduced a secondary composite cardiovascular end point, but also increased heart failure events and hospital admissions. ${ }^{9}$ In the ORIGIN trial, insulin glargine failed to reduce cardiovascular outcomes relative to placebo. ${ }^{10}$ More recently, dipeptidyl peptidase-4 (DPP-4) inhibitors have been shown to have a neutral cardiovascular effect (even when data were combined in a meta-analysis), except saxagliptin, which increased the risk of hospital admissions for heart failure. ${ }^{11}$ Glucagon-like peptide-1 (GLP-1) agonists have shown promise with respect to cardiovascular benefit, although the data so far are heterogeneous, and a practical barrier to uptake is the need for injection. In the LEADER trial, liraglutide reduced a composite cardiovascular end point and all-cause mortality. ${ }^{12}$ However, when data for multiple GLP-1 agonists were combined in a meta-analysis, there was a reduction in all-cause mortality, but not in cardiovascular death, 
nonfatal myocardial infarction, or nonfatal stroke. ${ }^{13}$ Finally, even metformin is fallible. In the UKPDS-34 trial, metformin reduced any diabetes-related end point and all-cause mortality when compared with dietary interventions in 753 overweight patients with type 2 diabetes. ${ }^{14}$ However, I would challenge proponents of metformin to apply the same rigorous critical appraisal to UKPDS-34 that we do for contemporary trials-would metformin still be considered first-line therapy if that trial were to be published today? Just because something was promising 20 years ago does not mean it remains relevant now. If that were true, we would all still be using Windows 98.

A recently published Bayesian hierarchical network metaanalysis of 236 randomized controlled trials (including EMPAREG OUTCOME and CANVAS) compared SGLT2 inhibitors, GLP-1 agonists, and DPP-4 inhibitors. ${ }^{15}$ Both the SGLT2 inhibitors and the GLP-1 agonists lowered all-cause and cardiovascular mortality relative to control (placebo or no treatment) and relative to DPP-4 inhibitors. Furthermore, the SGLT2 inhibitors were associated with lower rates of heart failure events and myocardial infarction relative to control.

Admittedly, more data are required to confirm (or potentially refute) the observed cardiovascular benefit of SGLT2 inhibitors. In this regard, at least 3 cardiovascular outcome trials are in progress: DECLARE-TIMI 58 with dapagliflozin, ${ }^{16}$ VERTIS with ertugliflozin, ${ }^{17}$ and SCORED with sotagliflozin. ${ }^{18}$

The SGLT2 inhibitors will continue to be aggressively marketed and prescribed, and thus it is imperative that clinicians understand the potential benefits and risks of therapy, so that they can help patients in making an informed decision. The observed benefit with SGLT2 inhibitors is encouraging, but has been limited to patients with, or at high risk of, cardiovascular disease. Additionally, long-term data are currently absent, and other practical barriers exist, such as cost. Pharmacovigilance is important, but I would caution readers not to dismiss the SGLT2 inhibitor data thus far, as they represent a potentially unparalleled advancement in the management of type 2 diabetes. Time will tell whether I am on the right side of history, but for now I would implore clinicians not to let apprehension bias your judgment. We should embrace SGLT2 inhibitors, albeit prudently, and avoid the temptation to pine for the "good old days" of treating type 2 diabetes with metformin and glyburide, just as we do not fondly reminisce about phenformin and chlorpropramide.

\section{References}

1. Guidance for industry: diabetes mellitus_evaluating cardiovascular risk in new antidiabetic therapies to treat type 2 diabetes. Silver Spring (MD): US Department of Health and Human Services, Food and Drug Administration, Center for Drug Evaluation and Research; 2008 [cited 21 May 2018]. Available from: https://www.fda.gov/downloads/Drugs/Guidances/ucm 071627.pdf

2. Zinman B, Wanner C, Lachin JM, Fitchett D, Bluhmki E, Hantel S, et al. Empagliflozin, cardiovascular outcomes, and mortality in type 2 diabetes. NEnglJ Med. 2015;373(22):2117-28.
3. Neal B, Perkovic V, Mahaffey KW, de Zeeuw D, Fulcher G, Erondu N, et al. Canagliflozin and cardiovascular and renal events in type 2 diabetes. N Engl J Med. 2017;377(7):644-57.

4. Riser Taylor S, Harris KB. The clinical efficacy and safety of sodium glucose cotransporter- 2 inhibitors in adults with type 2 diabetes mellitus. Pharmacotherapy. 2013;33(9):984-99.

5. Fitchett D, Zinman B, Wanner C, Lachin JM, Hantel S, Salsali A, et al. Heart failure outcomes with empagliflozin in patients with type 2 diabetes at high cardiovascular risk: results of the EMPA-REG OUTCOME® trial. Eur Heart J. 2016;37(19):1526-34.

6. Wanner C, Inzucchi SE, Lachin JM, Fitchett D, von Eynatten M, Mattheus $\mathrm{M}$, et al. Empagliflozin and progression of kidney disease in type 2 diabetes. N Engl J Med. 2016;375(4):323-34.

7. Abdelmoneim AS, Eurich DT, Light PE, Senior PA, Seubert JM, Makowsky MJ, et al. Cardiovascular safety of sulfonylureas: over 40 years of continuous controversy with an answer. Diabetes Obes Metab. 2015;17(6):523-32.

8. Nissen SE, Wolski K. Effect of rosiglitazone on the risk of myocardial infarction and death from cardiovascular causes. $N$ Engl J Med. 2007; 356(24):2457-71. Erratum in: N Engl J Med. 2007;357(1):100.

9. Dormandy JA, Charbonnel B, Eckland DJ, Erdmann E, Massi-Benedetti M, Moules IK, et al. Secondary prevention of macrovascular events in patients with type 2 diabetes in the PROactive Study (PROspective pioglitAzone Clinical Trial In macroVascular Events): a randomised controlled trial. Lancet. 2005;366(9493):1279-89.

10. ORIGIN trial investigators; Gerstain HC, Bosch J, Dagenais GR, Díaz R, Jung $\mathrm{H}$, Maggioni AP, et al. Basal insulin and cardiovascular and other outcomes in dysglycemia. N Engl J Med. 2012;367(4):319-28.

11. Barry AR, Turgeon RD. DPP-4 inhibitors: the Seinfeld of oral antihyperglycemics. Can J Hosp Pharm. 2016;69(3):253-4.

12. Marso SP, Daniels GH, Brown-Frandsen K, Kristensen P, Mann JFE, Nauck MA, et al. Liraglutide and cardiovascular outcomes in type 2 diabetes. NEngl J Med. 2016;375(4):311-22.

13. Peterson SC, Barry AR. Effect of glucagon-like peptide-1 receptor agonists on all-cause mortality and cardiovascular outcomes: a meta-analysis. Curr Diabetes Rev. 2018;14(3):273-9.

14. UK Prospective Diabetes Study (UKPDS) Group. Effect of intensive bloodglucose control with metformin on complications in overweight patients with type 2 diabetes (UKPDS-34). Lancet. 1998;352(9131):854-65.

15. Zheng SL, Roddick AJ, Aghar-Jaffar R, Shun-Shin MJ, Francis D, Oliver $\mathrm{N}$, et al. Association between use of sodium-glucose cotransporter 2 inhibitors, glucagon-like peptide 1 agonists, and dipeptidyl peptidase 4 inhibitors with all-cause mortality in patients with type 2 diabetes: a systematic review and meta-analysis. JAMA. 2018;319(15):1580-91.

16. Identifier NCT01730534: Multicenter trial to evaluate the effect of dapagliflozin on the incidence of cardiovascular events (DECLARETIMI58). In: Clinicialtrials.gov [database on internet]. Bethesda (MD): National Library of Medicine; 2012 Nov 21 [cited 2018 May 21]. Available from: https://clinicaltrials.gov/ct2/show/NCT01730534

17. Identifier NCT01986881: Cardiovascular outcomes following ertugliflozin treatment in type 2 diabetes mellitus participants with vascular disease, the VERTIS CV Study (MK-8835-004). In: Clinicialtrials.gov [database on internet]. Bethesda (MD): National Library of Medicine; 2013 Nov 19 [cited 2018 May 21]. Available from: https://clinicaltrials.gov/ct2/show/ NCT01986881

18. Identifier NCT03315143: Effect of sotagliflozin on cardiovascular and renal events in patients with type 2 diabetes and moderate renal impairment who are at cardiovascular risk (SCORED). In: Clinicialtrials.gov [database on internet]. Bethesda (MD): National Library of Medicine; 2017 Oct 19 [cited 2018 May 21]. Available from: https://clinicaltrials.gov/ct2/show/ NCT03315143

Arden R Barry, BSc, BSc(Pharm), PharmD, ACPR

Chilliwack General Hospital, Lower Mainland Pharmacy Services Chilliwack, British Columbia

Faculty of Pharmaceutical Sciences, University of British Columbia Vancouver, British Columbia

Competing interests: None declared. 


\section{THE "CON" SIDE}

Emphasis has been placed on the cardiovascular safety of antihyperglycemic drugs for type 2 diabetes mellitus since the release of a meta-analysis ${ }^{1}$ and randomized controlled trial ${ }^{2}$ that signalled increased risk of myocardial infarction and heart failure with rosiglitazone. Given that the ultimate goal of treating diabetes is the prevention of macrovascular and microvascular events, the results of those studies highlighted a disconnect between lowering glycated hemoglobin (A1C) and reducing the long-term complications of diabetes. How the $\mathrm{A} 1 \mathrm{C}$ is lowered appears more important than the A1C level itself. What has followed over the past decade is a disappointing series of trials evaluating the cardiovascular safety of a series of newer drugs for type 2 diabetes, most of which have found a "neutral" effect on major adverse cardiovascular events (MACE), despite these drugs having a positive effect on lowering A1C.3.7 With the publication of 2 trials for sodium glucose co-transporter 2 (SGLT2) inhibitors ${ }^{8,9}$ signalling a possible reduction in MACE, prescribers and guideline writers were quick to embrace adoption of the class as a preferred second-line drug (after metformin) in patients at high risk for cardiovascular events. ${ }^{10}$ However, a closer analysis of the trials and safety profile of these drugs indicates that caution should be exercised when considering their use.

In EMPA-REG OUTCOME, an industry-sponsored trial in which 7 employees of Boehringer Ingelheim were study authors, 7020 participants with type 2 diabetes were randomly assigned to receive empagliflozin $10 \mathrm{mg}$ or $25 \mathrm{mg}$ or placebo, and were followed for a median 3.1 years. ${ }^{8}$ At the end of the trial, the authors reported that there was a reduction in cardiovascular mortality (hazard ratio [HR] 0.62, $95 \%$ confidence interval [CI] 0.49-0.77) and hospital admissions due to heart failure (HR 0.65, 95\% CI 0.50-0.85). However, weaknesses in the trial methodology put these findings in serious doubt. The composite primary end point of MACE (HR $0.86,95 \%$ CI $0.74-0.99$ ) was driven almost exclusively by a statistically significant lower risk of cardiovascular deaths. ${ }^{8,11}$ Deaths ruled as "non-assessable" - accounting for $40 \%$ of all cardiovascular deaths analyzed — were presumed to be cardiovascular deaths. In a sensitivity analysis performed by the US Food and Drug Administration (FDA) that removed these deaths from the cardiovascular death analysis, empagliflozin was no longer superior to placebo for MACE (HR 0.90, 95\% CI 0.77-1.06). ${ }^{11}$ In the original study, silent myocardial infarctions were not independently assessed, and were not included in the primary composite outcome. However, when the FDA included these events in the primary outcome as part of another sensitivity analysis, the primary outcome was no longer statistically significant. The study also found a statistically significant reduction in hospital admissions due to heart failure. However, this outcome was not controlled for type I error, and the trial was not designed to assess it. As a result, critical information needed to confirm heart failure status was not collected. In addition, several modifications to the definition of hospital admission due to heart failure were also made over the course of the trial, which introduced substantial bias in the collection and analysis of heart failure outcomes. As a result of these issues with the adjudication of outcomes and the statistical analysis, no confidence can be placed in any observed differences between empagliflozin and placebo for the main cardiovascular outcomes.

In CANVAS, an industry-sponsored trial program in which 4 employees of Janssen were study authors, data from 10142 participants in 2 randomized controlled trials (CANVAS for cardiovascular outcomes and CANVAS-R for renal outcomes) were analyzed to assess the effect of canagliflozin $100 \mathrm{mg}$ or $300 \mathrm{mg}$ on cardiovascular outcomes relative to placebo. ${ }^{9}$ According to the initial protocol for CANVAS, the study was to enroll 4330 participants in the first phase of the trial, and if the cardiovascular protection and safety end points were met, a further 14000 participants were to be enrolled. ${ }^{12}$ However, after the initial unblinding of results in 2012, a decision was made not to continue with enrolment, but to open a new trial (CANVAS-R) and combine its results with those of CANVAS. As a result, CANVAS was not a single trial with a homogenous population, but was instead 2 separate trials involving 2 different populations, with differences in several aspects of the study design, including inclusion and exclusion criteria, dosing of canagliflozin, and primary objectives. CANVAS-R had an apparently sicker population than CANVAS, with higher event rates and larger observed reductions in the HRs for many cardiovascular outcomes. ${ }^{13}$ The fact that there were larger observed differences in some event rates in the second trial, after unblinding of data from the initial cohort, and subsequent modifications to trial design bring into question the amount of bias influencing the final outcome observed. In addition, the 2 cohorts had meaningful differences in follow-up: 295.9 weeks in CANVAS and 108.0 weeks in CANVAS-R.

The combined results from the 2 cohorts in CANVAS demonstrated a reduction of risk in a composite MACE end point (HR $0.86,95 \%$ CI $0.75-0.97$ ), with event rates of 26.9 versus 31.5 per 1000 patient-years. ${ }^{9}$ This finding was counterbalanced by a statistically significant increase in amputations, fractures, infections of male genitalia, mycotic genital infections in women, volume depletion, and osmotic diuresis. For every 1000 patient-years, 4.6 cardiovascular events will be prevented, but at the expense of causing 2.9 amputations and 3.5 fractures. There is some speculation that these risks are exclusively linked to canagliflozin, as they were not observed in EMPA-REG OUTCOME; however, data for these outcomes were systematically collected in CANVAS but not in EMPA-REG OUTCOME. A statistically significant reduction in cardiovascular mortality was not seen with canagliflozin, as it was with empagliflozin, which could reflect differences in the patient populations of the 2 studies or could further bring into question the validity of the EMPA-REG OUTCOME result.

The cardiovascular safety trial for dapagliflozin is still in progress, with planned completion later in 2018. ${ }^{14}$ Until then, we have to rely on surrogate $\mathrm{A} 1 \mathrm{C}$ data for direction. Results from network meta-analyses suggest that the A1C-lowering effect of dapagliflozin is less than that observed with others in the same class, and it has a 
reduced effect in patients with chronic kidney disease. ${ }^{15-17}$ Given that up to $60 \%$ of patients with type 2 diabetes will have this comorbidity, this limitation substantially narrows the population eligible for treatment. ${ }^{18}$

In conclusion, the incremental reduction in MACE observed with canagliflozin in a pooled analysis ${ }^{9}$ and the questionable result from a trial for empagliflozin that had low methodological quality ${ }^{8}$ do not suggest that SGLT2 inhibitors are a panacea for patients living with type 2 diabetes. The small benefit, if it truly exists, must be balanced against a well-documented list of harms that elevate risks of already common conditions in type 2 diabetes, including a nearequivalent increase in the risk of amputation and fracture. FDA advisories about ketoacidosis, urinary tract infections, acute kidney injury, fractures, and amputations should also be weighed in the decision to prescribe these drugs. ${ }^{19-22}$ Reduced efficacy or contraindication in later stages of chronic kidney disease substantially limits their use. We should also be concerned about the increase in infections in a population that is already prone to them. As we continue to learn more about this class of drugs, a healthy dose of skepticism should be prescribed when evaluating SGLT2 inhibitors for treating type 2 diabetes in patients with cardiovascular disease.

\section{References}

1. Nissen SE, Wolski K. Effect of rosiglitazone on the risk of myocardial infarction and death from cardiovascular causes. $N$ Engl J Med. 2007; 356(24):2457-71.

2. Home PD, Pocock SJ, Beck-Nielsen H, Curtis PS, Gomis R, Hanefeld M, et al. Rosiglitazone evaluated for cardiovascular outcomes in oral agent combination therapy for type 2 diabetes (RECORD): a multicentre, randomised, open-label trial. Lancet. 2009;373(9681):2125-35.

3. Dormandy JA, Charbonnel B, Eckland DJ, Erdmann E, Massi-Benedetti $\mathrm{M}$, Moules IK, et al. Secondary prevention of macrovascular events in patients with type 2 diabetes in the PROactive Study (PROspective pioglitAzone Clinical Trial In macroVascular Events): a randomised controlled trial. Lancet. 2005;366(9493):1279-89.

4. Scirica BM, Bhatt DL, Braunwald E, Steg PG, Davidson J, Hirshberg B, et al. Saxagliptin and cardiovascular outcomes in patients with type 2 diabetes mellitus. NEngl J Med. 2013;369(14):1317-26.

5. White WB, Cannon CP, Heller SR, Nissen SE, Bergenstal RM, Bakris GL, et al. Alogliptin after acute coronary syndrome in patients with type 2 diabetes. N Engl J Med. 2013;369(14):1327-35.

6. Green JB, Bethel MA, Armstrong PW, Buse JB, Engel SS, Garg J, et al. Effect of sitagliptin on cardiovascular outcomes in type 2 diabetes. N Engl J Med. 2015;373(3):232-42.

7. Pfeffer MA, Claggett B, Diaz R, Dickstein K, Gersein HC, Køber LV, et al. Lixisenatide in patients with type 2 diabetes and acute coronary syndrome. N Engl J Med. 2015;373(23):2247-57.

8. Zinman B, Wanner C, Lachin JM, Fitchett D, Bluhmki E, Hantel S, et al. Empagliflozin, cardiovascular outcomes, and mortality in type 2 diabetes. NEngl J Med. 2015;373(22):2117-28.

9. Neal B, Perkovic V, Mahaffey KW, de Zeeuw D, Fulcher G, Erondu N, et al. Canagliflozin and cardiovascular and renal events in type 2 diabetes. NEngl J Med. 2017;377(7):644-57.

10. 2018 Clinical Practice Guidelines Committees. Diabetes Canada 2018 clinical practice guidelines for the prevention and management of diabetes in Canada. Can J Diabetes. 2018;42 Suppl 1:S1-S325.

11. FDA briefing document: Endocrine and Metabolic Drug Advisory Committee Meeting: empagloflozin (JARDIANCE) tablets and empagliflozin and metformin hydrochloride (SYNJARDY) tablets. Silver Spring (MD): US Food and Drug Administration; 2016 Jun 28 [cited 2018 May 17]. Available from: https://www.fda.gov/downloads/AdvisoryCommittees/ UCM508422.pdf

12. Protocol for: Neal B, Perkovic V, Mahaffey KW, de Zeeuw D, Fulcher G, Erondu N, et al. Canagliflozin and cardiovascular and renal events in type 2 diabetes. $N$ Engl J Med. 2017;377(7):644-57. Available from: https:// www.nejm.org/doi/suppl/10.1056/NEJMoa1611925/suppl_file/nejmoa161 1925_protocol.pdf [cited 2018 May 17].

13. Supplement to: Neal B, Perkovic V, Mahaffey KW, de Zeeuw D, Fulcher G, Erondu N, et al. Canagliflozin and cardiovascular and renal events in type 2 diabetes. N Engl J Med. 2017;377(7):644-57. Available from: https:// www.nejm.org/doi/suppl/10.1056/NEJMoa1611925/suppl_file/nejmoa161 1925_appendix.pdf [cited 2018 May 17].

14. Identifier NCT01730534: Multicenter trial to evaluate the effect of dapagliflozin on the incidence of cardiovascular events (DECLARE-TIMI58). In: ClinicalTrials.gov [database on internet]. Bethesda (MD): National Library of Medicine; 2012 Nov 21 [cited 2018 May 17]. Available from: https://clinicaltrials.gov/ct2/show/NCT01730534

15. Storgaard H, Gluud LL, Bennett C, Grøndahl MF, Christensen MB, Knop FK, et al. Benefits and harms of sodium-glucose co-transporter 2 inhibitors in patients with type 2 diabetes: a systematic review and meta-analysis. PLoS One. 2016;11(11): e0166125.

16. Shyangdan DS, Uthman OA, Waugh N. SGLT-2 receptor inhibitors for treating patients with type 2 diabetes mellitus: a systematic review and network meta-analysis. BMJ Open. 2016;6(2):e009417.

17. Dekkers CCJ, Wheeler DC, Sjöström CD, Stefansson BV, Cain V, Heerspink HJL. Effects of the sodium-glucose co-transporter 2 inhibitor dapagliflozin in patients with type 2 diabetes and stages $3 \mathrm{~b}-4$ chronic kidney disease. Nephrol Dial Transplant. 2018;33(7):1280.

18. Wu B, Bell K, Stanford A, Kern DM, Tunceli O, Vupputuri S, et al. Understanding CKD among patients with T2DM: prevalence, temporal trends, and treatment patterns_-NHANES 2007-2012. BMJ Open Diabet Res Care. 2016;4(1):e000154.

19. FDA drug safety communication: FDA revises label of diabetes drug canagliflozin (Invokana, Invokamet) to include updates on bone fracture risk and new information on decreased bone mineral density. Silver Spring (MD): US Food and Drug Administration; 2015 Oct 9 [cited 2018 May 17]. Available from: https://www.fda.gov/Drugs/DrugSafety/ucm461449.htm

20. FDA drug safety communication: FDA revises labels of SGLT2 inhibitors for diabetes to include warnings about too much acid in the blood and serious urinary tract infections. Silver Spring (MD): US Food and Drug Administration; 2015 Apr 12 [cited 2018 May 17]. Available from: https://www.fda.gov/Drugs/DrugSafety/ucm475463.htm

21. FDA drug safety communication: FDA confirms increased risk of leg and foot amputations with the diabetes medicine canagliflozin (Invokana, Invokamet, Invokamet XR). Silver Spring (MD): US Food and Drug Administration; 2017 May 16 [cited 2018 May 17]. Available from: https://www.fda.gov/ Drugs/DrugSafety/ucm557507.htm

22. FDA drug safety communication: FDA strengthens kidney warnings for diabetes medicines canagliflozin (Invokana, Invokamet) and dapagliflozin (Farxiga, Xigduo XR). Silver Spring (MD): US Food and Drug Administration; 2016 Jun 14 [cited 2018 May 17]. Available from: https://www.fda.gov/ Drugs/DrugSafety/ucm505860.htm

Bradley R Mitchelmore, BSc(Pharm), ACPR, PharmD

Rideau Community Health Services

Merrickville, Ontario

Competing interests: Bradley Mitchelmore is a former employee of the Canadian Agency for Drugs and Technologies in Health (CADTH), which provides reimbursement recommendations to 18 public drug plans in Canada. This organization has previously reviewed empagliflozin, canagliflozin, and dapagliflozin for multiple indications in type 2 diabetes. The Canadian Drug Expert Committee, part of CADTH, has provided "reimburse with clinical criteria and/or conditions" recommendations for all 3 drugs for various indications, including a recommendation to "reimburse with clinical criteria and/or conditions" for empaglifozin to reduce the incidence of cardiovascular death. Of all indications reviewed for this class of drugs, CADTH recommended a "do not reimburse" recommendation only for dapagliflozin as triple therapy with metformin and a sulfonylurea. 